\section{OP0079 LIMB GIRDLE MUSCULAR DYSTROPHY TYPE 2B - A RARE MYOSITIS MIMIC}

A. Merriman ${ }^{1}$, S. Boyle ${ }^{1} .{ }^{1}$ Western General Hospital, Rheumatic Diseases Unit, Edinburgh, United Kingdom

Background: Proximal muscle weakness with associated raised creatine kinase (CK) commonly leads to referral to Rheumatology for the investigation of Idiopathic Inflammatory Myopathy (IIM). Some genetic myopathies can have a similar presentation with investigations that suggest inflammatory disease, leading to difficulty with accurate diagnosis (Amato \& Brown, 2011; Harlan \& Mammen, 2019).

Objectives: To describe the case of a patient with Limb Girdle Muscular Dystrophy Type 2B (LGMD2B), whose initial presentation mimicked an inflammatory myopathy.

Methods: Case report

Results: A 43-year-old patient was reviewed by Rheumatology due to proximal muscle weakness with a raised CK. Muscle biopsy was suggestive of inflammatory myopathy. Therefore, he was started on treatment with corticosteroids. Corticosteroid treatment resulted in no improvement in his weakness or CK. His diagnosis was reviewed, and he was referred to the Neurology and Genetics services. Following molecular genetic analysis, a diagnosis of Limb Girdle Muscular Dystrophy Type 2B was made.

Conclusion: Muscle biopsies can suggest an inflammatory aetiology in some genetic myopathies (Amato \& Brown, 2011; Harlan \& Mammen, 2019). If a patient with suspected IIM presents with atypical features, or they do not respond as expected to treatment, then consider a genetic myopathy such as LGMD2B as a cause and involve the Neurology and Genetics services in the case.

REFERENCES:

[1] Amato, A. A., \& Brown, R. H., Jr. (2011). Dysferlinopathies. Handb Clin Neurol, 101, 111-118. https://doi.org/10.1016/b978-0-08-045031-5.00007-4

[2] Harlan, M \& Mammen A. L (2019). Myositis Mimics: The Differential Diagnosis of Myositis. In: R. Aggarwal and C. V. Oddis (Eds.) Managing Myositis (pp. 209-223). Springer, Cham.

Disclosure of Interests: None declared

DOI: 10.1136/annrheumdis-2021-eular.1251

\section{OP0080 OSTEOPOIKILOSIS IN PATIENT WITH ANKYLOSING SPONDYLITIS}

V. Omelchenko ${ }^{1,2}$, E. Letyagina $^{1,2}$, Y. Kurochkina ${ }^{1}$, A. Akimova ${ }^{1}$, M. Korolev $^{1,2}$ ${ }^{1}$ RICEL - Branch of IC\&G SB RAS, Rheumatology Department, Novosibirsk, Russian Federation; ${ }^{2}$ Novosibirsk State University, Internal Medicine Section, Novosibirsk, Russian Federation

Background: Osteopoikilosis (OPK) - a rare congenital genetic-mediated benign sclerosing disease of skeleton. OPK is usually clinically asymptomatic and is most often an incidental finding during an X-ray study [1]. The lesions are from 2 to $10 \mathrm{~mm}$ and are located in the area of the ischial, pubic bones, metaepiphyseal regions of the tubular bones. The axial skeleton is usually not affected in OPK. As early described, OPK may be associated with inflammatory rheumatic diseases, such as rheumatoid arthritis, spondyloarthritis, reactive arthritis and scleroderma [2-4]. Ankylosing spondylitis is a chronic rheumatic disease from the group of spondyloarthritis with obligatory involvement of the sacroiliac joints and frequent involvement of entheses and peripheral joints. Although a significant mutual influence of OPK and AS has not been proven, their combination can lead to unreasonable over-examination in real clinical practice.

Objectives: To present a clinical case of rare coexisting of osteopoikilosis and ankylosing spondylitis.

Methods: Case report. The patient was treated and examined based on real clinical practice.

Results: A 33-year-old man was admitted to our rheumatology department with a three-year history of inflammation pain in cervical region of spine and hip, knees and shoulder joints and long morning stiffness duration (up to 3 hours). AS was earlier verified based on modified New York criteria (1984) and Nonsteroidal anti-inflammatory drugs were prescribed promptly. On the outpatient basis he also received sulfasalazine and methotrexate due to arthritis and enthesitis. Nevertheless he had high disease activity (ESR $66 \mathrm{~mm} / \mathrm{h}$, CRP $77.5 \mathrm{mg} / \mathrm{l}$, BAS$\mathrm{DAl}=7.5$ ), severely limited range of motion in the cervical, thoracic and lumbar spine, arthritis, enthesitis when he entered in our clinic. He had been treated with secukinumab $150 \mathrm{mg}$ for fourteen months and then he was switched to golimumab $50 \mathrm{mg}$ per month because of lack of efficacy. Low disease activity has now been achieved.
During the assessment of radiographs of the pelvic bones and spine, typical changes of the late stage of ankylosing spondylitis (syndesmophytes, stage 4 sacroilitis) were revealed at the first visit in our clinic. At the same time, a number of phenomena were discovered that were not related to the course of the AS, which required expanding the differential diagnosis range. The tumor process and endocrine pathologies were excluded. OPK was diagnosed on the basis of characteristic changes in various parts of the skeleton, especially in phalanges, wrists and pelvis bones (fig.1).

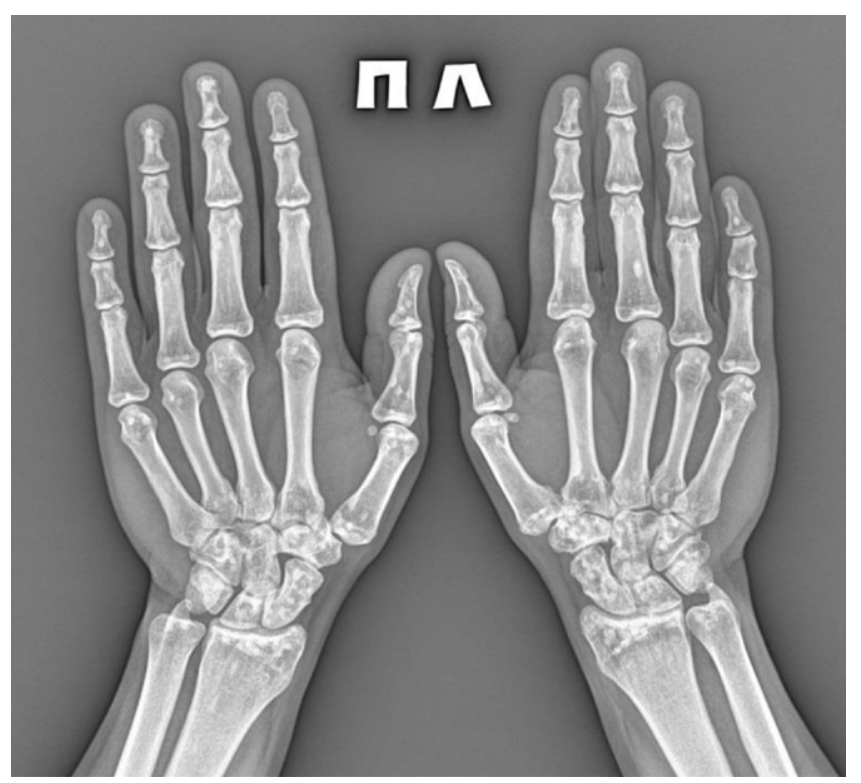

Figure 1. OPK spots in phalanges and wrists.

Conclusion: We've reported rare case of AS and OPK coexisting in young patient. The identification of typical radiological phenomena and correct evaluation of clinical data may allow to avoid unnecessary expensive examinations. REFERENCES:

[1] Carpintero P. et al. Clinical features of ten cases of osteopoikilosis // Clin Rheumatol. 2004. Vol. 23, № 6. P. 505-508.

[2] Joseph R.O., Thomas J., Shenoy P. Osteopoikilosis and ankylosing spondylitis: Strange bedfellows: A case report // Int. J. Rheum. Dis. 2019. Vol. 22, № 6. P. $1162-1164$.

[3] Akgul O. et al. Osteopoikilosis and ankylosing spondylitis: A rare coexistence and good response to TNF blocker adalimumab // Int. J. Rheum. Dis. 2015 Vol. 18, № 3. P. 372-374.

[4]Ruaro B. et al. Coexistence of osteopoikilosis with seronegative spondyloarthritis and Raynaud's phenomenon: first case report with evaluation of the nailfold capillary bed and literature review. // Reumatismo. 2012. Vol. 64, № 5. P. 335-339.

Disclosure of Interests: None declared

DOI: 10.1136/annrheumdis-2021-eular.2267

\section{OP0081 \\ A CASE OF ATYPICAL MYCOBACTERIUM INFECTION COMPLICATING EXTRA-NODAL ROSAI-DORFMAN DISEASE IN A PATIENT WITH SYSTEMIC LUPUS ERYTHEMATOSUS (SLE)}

K. Su ${ }^{1}$, G. Hagan ${ }^{2}$, S. Tosounidou ${ }^{1}$, C. Gordon ${ }^{1,3}$, J. Reynolds ${ }^{1,3} .{ }^{1}$ Sandwell and West Birmingham NHS Trust, Rheumatology, Birmingham, United Kingdom;

${ }^{2}$ Sandwell and West Birmingham NHS Trust, Respiratory, Birmingham, United Kingdom; ${ }^{3}$ University of Birmingham, Institute of Inflammation and Aging, Birmingham, United Kingdom

Background: We present the case of a 28 year old Black-British female with severe SLE requiring treatment with rituximab in 2012 due to persistent low-grade activity and severe episodes of pleuro-pericardial effusions, pancytopaenia, fever and weight loss 
Her other background includes beta-thalassaemia trait and excision of calcific fibrotic tissue on bilateral anterolateral orbits in 2015

In 2018 she reported an 18-month history of non-tender, non-fluctuant, slow growing left thigh mass with USS revealing a well demarcated subcutaneous complex cystic lesion of $\sim 2 \times 4 \times 7 \mathrm{~cm}$. There was no preceding trauma or skin infection. Histology from a needle biopsy revealed diffuse histiocytosis with positive immunohistochemistry $(\mathrm{ICH})$ for S100, CD68 and CD31, it was negative for CD1a, consistent with Extra-nodal Rosai-Dorfman disease (RDD).

She developed constitutional symptoms after reporting months of gradual weight loss with gradual ESR, CRP rise and leucocytosis. Her SLE symptoms were stable and given lack of SLE-specific symptoms; PET-CT was used to identify systemic RDD; the thigh mass showed strong FDG avidity along with a small focus of uptake in the small bowel, thought to be RDD related with no other areas of uptake.

She had ongoing ooze from the enlarging thigh lesion ( $5 \times 26 \times 15 \mathrm{~cm})$, this was sent for MCS and AAFB; which isolated Mycobacterium avium. She was treated with rifampicin, ethambutol and clarithromycin resulting in improved thigh lesion, constitutional symptoms and inflammatory markers.

Objectives:

[1] To describe a rare associated complication of severe SLE and to educate and inform clinicians regarding possible masquerades of disease

[2] To education and inform about the approach to diagnosis of mycobacterium infection.

Methods: Case report and literature review.

Results: Mycobacterium infections rarely complicate RDD; to date, only one case report is published involving an HIV infected patient with RDD confirmed on LN biopsy presenting with splenomegaly and treated with oral corticosteroids (OCS) complicated by Mycobacterium avium complex and Salmonella enterica confirmed on bone marrow biopsy/culture, similar to our patient, he presented with constitutional symptoms and weight $\operatorname{loss}^{(2)}$

Mycobacterium can also mimic RDD, a case report has described a 74 year old with tender lymphadenopathy diagnosed with RDD on LN biopsy. She was treated with IV and OCS, but was unresponsive. A repeat LN biopsy and CT imaging revealed the presence of mycobacterium kansasii; her biopsy was positive for CD68/S100 throughout. Of note, she had high levels of anti-interferon autoantibodies and was diagnosed with adult-onset immunodeficiency syndrome ${ }^{(3)}$

Conclusion: This case illustrates the need for a MDT approach for multi-system diseases such as SLE and RDD, and the need to consider atypical infections when blood tests are incongruent with clinical state.

REFERENCES:

[1] Bruce-Brand C, Schneider JW, Schubert P. Rosai-Dorfman disease: an overview. J Clin Pathol. 2020 Nov;73(11):697-705. doi: 10.1136/jclinpath-2020-206733.

[2] Olmedo-Reneaum A, Molina-Jaimes A, Conde-Vazquez E, Montero-Vazquez S. Rosai-Dorfman disease and superinfection due to Salmonella enterica and Mycobacterium avium complex in a patient living with HIV. IDCases. 2020 Jan 14;19:e00698.

[3] King YA, Hu CH, Lee YJ, Lin CF, Liu D, Wang KH. Disseminated cutaneous Mycobacterium kansasii infection presenting with Rosai-Dorfman disease-like histological features in a patient carrying anti-interferon- $\gamma$ autoantibodies. $J$ Dermatol. 2017 Dec;44(12):1396-1400.
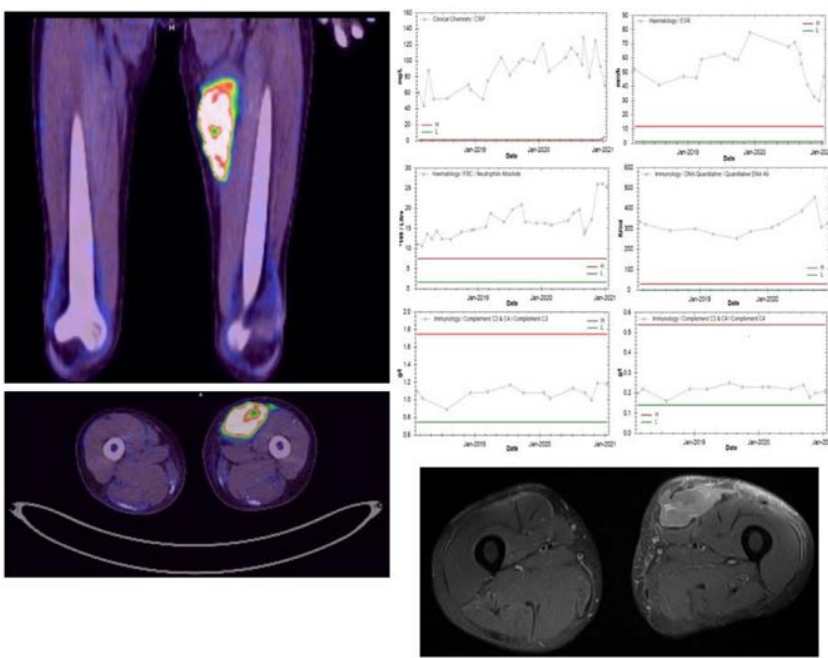

Image 1.
Table 1.

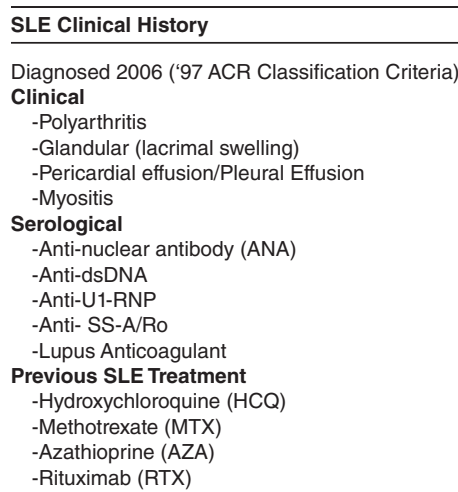

Disclosure of Interests: None declared

DOI: 10.1136/annrheumdis-2021-eular.2415

\section{Challenges for Patients and Patients' Organisations in Times of the Pandemic}

\section{OP0082-PARE THE EFFECT COVID-19 HAS ON THE MENTAL HEALTH OF PEOPLE LIVING WITH RHEUMATIC DISEASES. FROM DATA TO INTERVENTIONS.}

S. Mingolla ${ }^{1}$, A. Celano ${ }^{2}$, M. Santopietro ${ }^{3} .{ }^{1}$ APMARR APS, Communication, Lecce IPuglia, Italy; ${ }^{2}$ APMARR APS, National Association of People with Rheumatic and Rare Diseases, Lecce, Italy; ${ }^{3}$ WeResearch, Ricerche di Marketing, Milan, Italy

Background: Covid-19 has had an important impact on the mental health conditions of over 5 million Italians suffering from one of the over 150 rheumatic diseases. In order to understand the psychological impact of the Covid-19 emergency and the restrictions imposed to counter it, the Italian National Association of People with Rheumatic and Rare Diseases - APMARR APS launched the research "Living with a rheumatic pathology".

Objectives: Gather data directly from Italian patients about the impact of the COVID-19 and consequent restrictions on their mental health and feelings; evaluate the most effective intervention to be implemented to face the pandemic by Patients organization.

Methods: A qualitative-quantitative survey was carried out through a questionnaire administered throughout the national territory to a sample of $N=1,001$ people. The people invited to complete the questionnaire were women $(55,9 \%)$ and men (44,1\%), aged $18-85$ years (age $18-41=26,7 \%$; age $42-65=64 \%$; age $>65=9,3 \%$ ) with at least one rheumatic pathology. The questionnaire was made up of 39 questions, of which 29 were closed and 10 were open. For the administration of the questionnaires, the CAWI (Computer Aided Web Interview) methodology of on-line survey was used. The 1,001 interviews were carried out from 7 to 14 August 2020.

Results: More than 4 out of 10 people (total sample $44.2 \%$; male $60 \%$, female $35,7 \%$; age $18-41=39,1 \%$; age $42-65=45,9 \%$; age $>65=50 \%$ ) declared that the emergency period has somehow caused a worsening of their health condition. People declared that the deterioration of their health is due to the emergency period for the following reasons: 1) Psychological: such as stress and anxiety: "Too much stress and anxiety made the symptoms worse."; "The stress of the quarantine affected my problem"; "Insomnia. Nervousness. General ailments. Depression. Strong stress" 2) Inability to perform physiotherapy and motor activities due to the lockdown 3) Postponement of examinations, visits and checks 4) remote working, in some cases described as harmful for people's mental and physical health: "Due to Covid19 I had to do remote working and I worked even 12 hours a day including holidays to the detriment of my family life".

Furthermore, from January 31, 2020 a significant increase emerged in communication problems with rheumatology specialist compared to the period before the emergency due to Covid-19. The sharp increase may be due to the situation of severe psychological stress to which also the doctors were subjected in the emergency phase: people could not find the comfort of being empathically listened to. 